\title{
Correction to: Optimising protocols for high-definition imaging of historic shipwrecks using multibeam echosounder
}

\author{
Kieran Westley ${ }^{1}$ (ID $\cdot$ Ruth Plets $^{1} \cdot$ Rory Quinn $^{1} \cdot$ Chris McGonigle $^{1} \cdot$ Fabio Sacchetti $^{2}$. \\ Mekayla Dale $^{1,2} \cdot$ Rory McNeary ${ }^{3} \cdot$ Annika Clements $^{4}$ \\ Published online: 4 May 2019 \\ (C) Springer-Verlag GmbH Germany, part of Springer Nature 2019
}

\section{Correction to: Archaeological and Anthropological Sciences https://doi.org/10.1007/s12520-019-00831-6}

The original version of this article, unfortunately, contained error. Additional correction in Table 1: please delete (') in the second column heading was incorrectly carried out. It should read "Acquisition settings test"; by this, the author meant only to delete the apostrophe symbol. However the typesetter has deleted the entire second column heading (i.e. in the online proof it read: "Acquisition settings' test ", but the corrected version should have read "Acquisition settings test". Given in the article is the correct table.

The original article has been corrected.

Publisher's note Springer Nature remains neutral with regard to jurisdictional claims in published maps and institutional affiliations.

The online version of the original article can be found at https://doi.org/ 10.1007/s12520-019-00831-6

Kieran Westley

kl.westley@ulster.ac.uk

1 School of Geography \& Environmental Sciences, Ulster University, Coleraine, Northern Ireland BT52 1SA, UK

2 Marine Institute, Oranmore, Co. Galway H91 R673, Republic of Ireland

3 Historic Environment Division, Department for Communities, Causeway Exchange, 1-7 Bedford Street, Belfast, Northern Ireland BT2 7EG, UK

4 Agri-Food and Biosciences Institute, Fisheries and Aquatic Ecosystems Branch, Newforge Lane, Belfast, Northern Ireland BT9 $5 \mathrm{PX}, \mathrm{UK}$ 
Table 1 Acquisition settings tested over SS Polwell

\begin{tabular}{|c|c|c|}
\hline Parameter & Acquisition settings test & Survey design test \\
\hline Frequency & Varied: $200 \mathrm{kHz}, 300 \mathrm{kHz}, 400 \mathrm{kHz}$ & Constant: $400 \mathrm{kHz}$ \\
\hline Pulse rate & Varied: $4 \mathrm{~Hz}, 8 \mathrm{~Hz}, 12 \mathrm{~Hz}$ & Constant: maximum (up to $50 \mathrm{~Hz}$ ) \\
\hline Angular sector (overall and port/starboard) & $\begin{array}{l}\text { Varied: } 40^{\circ}\left(20^{\circ} / 20^{\circ}\right), 60^{\circ}\left(30^{\circ} / 30^{\circ}\right) \text { and }\left(0^{\circ} / 60^{\circ}\right), 80^{\circ} \\
\quad\left(40^{\circ}, 40^{\circ}\right)\end{array}$ & Varied: $110^{\circ}\left(55^{\circ} / 55^{\circ}\right), 60^{\circ}\left(30^{\circ} / 30^{\circ}\right)$ \\
\hline Sector mode & Constant: Normal (three sectors) & $\begin{array}{l}\text { Varied: Normal (three sectors), single sector } \\
\quad \text { (only middle sector) }\end{array}$ \\
\hline Beam spacing & Constant: High Density Equidistant & Constant: High Density Equidistant \\
\hline $\begin{array}{l}\text { Pulse length (lower values for single sector, higher } \\
\text { for normal sector at a given frequency) }\end{array}$ & $\begin{array}{l}\text { Constant: Short CW }(200 \mathrm{kHz}=70 \mu \mathrm{s} ; 300 \mathrm{kHz} \\
\quad=70 \mu \mathrm{s} ; 400 \mathrm{kHz}=50 \mu \mathrm{s})\end{array}$ & $\begin{array}{l}\text { Constant: Short CW }(200 \mathrm{kHz}=35-70 \mu \mathrm{s} ; \\
\quad 300 \mathrm{kHz}=35-70 \mu \mathrm{s} ; 400 \mathrm{kHz}=25-50 \mu \mathrm{s})\end{array}$ \\
\hline Bottom detection & Varied: Normal, minimum depth and tracking & Constant: minimum depth \\
\hline
\end{tabular}

